\title{
"Later, lazier, and unluckier": a heuristic profile of high vulnerability is an independent predictor of uncontrolled blood pressure (the PREVIEW study)
}

\author{
This article was published in the following Dove Press journal: \\ International Journal of General Medicine \\ 18 June 2010 \\ Number of times this article has been viewed
}

\author{
Ivo Abraham ${ }^{1,2}$ \\ Christopher Lee ${ }^{1,2}$ \\ MinKyoung Song 1,3 \\ Stefaan Vancayzeele ${ }^{4}$ \\ Heidi Brié ${ }^{4}$ \\ Christine Hermans ${ }^{4}$ \\ Patricia Van der Niepen ${ }^{5}$ \\ Karen MacDonald ${ }^{2}$ \\ 'University of Arizona, Tucson, AZ, \\ USA; ${ }^{2}$ Matrix45, Earlysville, VA, USA; \\ ${ }^{3} \mathrm{School}$ of Nursing, University of \\ Pennsylvania, Philadelphia, PA, USA; \\ ${ }^{4}$ Department of Nephrology and \\ Hypertension, Universitair Ziekenhuis \\ Brussel, Vrije Universiteit Brussel, \\ Brussels, Belgium; ${ }^{5}$ Novartis Pharma, \\ Vilvoorde, Belgium
}

Correspondence: Ivo Abraham 620 Frays Ridge Road, Earlysville, VA 22936, USA

Tel +I 434978 I045

Fax +I 9789458374

Email iabraham@matrix45.com
Objective: Vulnerability profiling, an alternative to deterministic risk assessment, offers clinicians a more intuitive but empirically-grounded assessment of patient risk. This study aimed to determine whether a heuristic profile of high vulnerability is an independent predictor of uncontrolled hypertension.

Methods: Secondary analysis of prospective observational study data on 2999 hypertensive patients treated with valsartan. Predictive validity of vulnerability profiling for first-line, secondline, and first-or-second-line antihypertensive treatment was inferred from 1) logistic regression models with adequate statistical fit, 2) statistically significant odds ratios for uncontrolled BP for the high-vulnerability cluster exceeding 1.00, and 3) correct classification rates for patients' BP control status.

Results: All models of uncontrolled BP were significant $(P<0.001)$; all odds ratios for the high-vulnerability cluster were greater than 1.00 and significant $(P<0.001)$. Correct classification rates for the highly-vulnerability cluster on uncontrolled BP after first-line, second-line, or either treatment were $91.1 \%, 61.2 \%$, and $93.5 \%$ for systolic BP; $74.5 \%, 65.8 \%$, and $76.7 \%$ for diastolic BP; and 92.8\%, 65.3\%, and 94.6\% for combined systolic and diastolic BP.

Conclusion: The heuristic profile of "later, lazier, and unluckier" is an intuitive and valid tool to help identify patients at greater risk for poor BP control seen in general practice.

Keywords: hypertension, heuristics, vulnerability, profiling, risk

\section{Introduction}

Although different formal algorithms to determine cardiovascular outcomes are available (eg, Framingham risk score ${ }^{1}$ and SCORE project $^{2}$ ), these tools are seldom used in primary care. Because of the required data collection, calculations, and time demands, as well as the deterministic nature of the results, using such algorithms rarely fits into the clinical flow of primary care encounters. The implicit prescriptive nature of these algorithms and that they provide a probabilistic assessment of comparable patients may further limit their utility in planning individual patient care. Moreover, deterministic systems can be criticized for discounting clinicians' expertise in evaluating patients and assessing risk. Thus, clinically-intuitive methods for identifying patients at greater risk are important, particularly in control of hypertension, in which blood pressure (BP) targets are seldom reached ${ }^{3-6}$ and patients are at high-risk for target organ damage. . $^{7}$ 
We recently reported on determinants of BP outcomes and control after 90 days of second-line treatment with the angiotensin II receptor blocker valsartan. ${ }^{9}$ The PREVIEW study was an observational trial involving 3194 patients in whom first-line treatment failed or was not tolerated. We used hierarchical cluster analysis to identify sub-cohorts of patients with differential vulnerability to uncontrolled hypertension. Cluster analysis, a subclass of data-mining, ${ }^{10}$ was used to discover latent patterns of subject similarity based on factors identified from the literature and clinical experience. ${ }^{11,12}$ We identified two clusters of patients with differential profiles of vulnerability to poor antihypertensive treatment outcomes.

As shown in Table 1, the highly-vulnerable cluster (HVC) of patients ( $n=1063$ or $35.4 \%$ ) differed from the remaining vulnerable cluster (VC) of patients by: a) having been diagnosed relatively late and with more severe hypertension, b) weighing and drinking more, and exercising less, c) presenting with greater general, cardiovascular, and renal/endocrine risk and more comorbid cardiovascular disease, d) having a family history of premature cardiovascular disease, and e) having a poor treatment response. Clinically, HVC patients had a higher propensity to be (only with helpful clinical mnemonics in mind, and without any derogatory intent) "later, lazier, and unluckier." In the PREVIEW study, the vulnerability clusters were determinants of both BP values and BP control as defined by the JNC-7/ESH-ESC ${ }^{13,14}$ guidelines (140/90 mmHg; for diabetics: $130 / 80 \mathrm{mmHg}$ ) in multivariate models. ${ }^{9}$
These initial aggregate findings indicate that being a HVC patient is predictive of BP outcomes in multivariate models that include other determinants. However, in order to be able to recommend the HVC profile as a means of identifying patients who may not respond to antihypertensive treatment, the profile must be further validated. This requires determining whether being a HVC patient is by and of itself a predictor of uncontrolled systolic BP (SBP), diastolic BP (DBP), and combined SBP/DBP - in the absence of other determinants. Further, the original analysis was limited to second-line treatment. It is also important to examine whether the HVC profile is predictive of uncontrolled BP after first-line treatment. These additional statistical analyses, which we report on in this present paper, are essential to determining the validity of the HVC profile as a predictor of poor response to antihypertensive treatment. Thus we examined the extent to which the HVC profile is an independent predictor of uncontrolled BP after first-line treatment (at physicians' clinical discretion), after second-line treatment with valsartan, and after either first- or second-line treatment.

\section{Methods \\ Procedures}

Procedures for sampling, variables and measurements, data collection and management, statistical analysis, and management of confounding variables are described in detail in the referent article. ${ }^{9}$

Table I Profiling of patients' vulnerability to uncontrolled hypertension

\begin{tabular}{|c|c|c|c|}
\hline & $\begin{array}{l}\text { Vulnerable patients } \\
(\mathrm{n}=1936)\end{array}$ & $\begin{array}{l}\text { Highly-vulnerable patients } \\
(\mathrm{n}=1063)\end{array}$ & \\
\hline & Mean ( $( \pm S D)$ & Mean ( $($ SD) & $P$-value \\
\hline SBP when diagnosed & $159.49( \pm 9.33)$ & $182.13( \pm 14.20)$ & $<0.001$ \\
\hline DBP when diagnosed & $94.07( \pm 6.90)$ & $104.12( \pm 11.38)$ & $<0.001$ \\
\hline General risk $^{\mathrm{a}}$ & $2.4060( \pm 1.54)$ & $2.6058( \pm 1.55)$ & 0.001 \\
\hline Cardiovascular risk ${ }^{\mathrm{b}}$ & $0.64 I 0( \pm I . I 2)$ & $0.7846( \pm \mathrm{I} .26)$ & 0.002 \\
\hline \multirow[t]{2}{*}{ Renal/endocrine risk ${ }^{c}$} & $0.1865( \pm .061)$ & $0.2389( \pm .067)$ & 0.038 \\
\hline & $\%(95 \% \mathrm{Cl})$ & $\%(95 \% \mathrm{Cl})$ & $P$-value \\
\hline Left ventricular hypertrophy & $11.4 \%(10.3-12.5)$ & $16.8 \%(15.6-18.0)$ & $<0.001$ \\
\hline Excessive alcohol use & $16.0 \%(\mid 4.9-17.1)$ & $18.9 \%(17.6-20.2)$ & 0.023 \\
\hline Lack of exercise & $55.8 \%(54.2-57.4)$ & $61.1 \%(59.5-62.7)$ & 0.005 \\
\hline Obesity & $42.5 \%(40.9-44.1)$ & $48.4 \%(46.7-50.1)$ & 0.001 \\
\hline Family history of early cardiovascular disease & $16.6 \%(15.4-17.8)$ & $19.5 \%(18.2-20.8)$ & 0.027 \\
\hline SBP controlled at start of treatment & $10.4 \%(9.4-11.4)$ & $6.1 \%(5.3-6.9)$ & $<0.001$ \\
\hline DBP controlled at start of treatment & $27.9 \%(26.4-29.4)$ & $21.3 \%(19.9-22.7)$ & $<0.001$ \\
\hline
\end{tabular}

Notes: Data adapted from Van der Niepen et al. ${ }^{9}$ Composite score of occurrence of hypercholesterolemia, diabetes mellitus, smoking, excess alcohol use, lack of regular physical exercise, obesity, advanced retinopathy; ' ${ }^{b}$ Composite score of occurrence of myocardial infarction, angina, coronary revascularization, left ventricular hypertrophy, ischemic and or hemorrhagic cerebrovascular accident, transient ischemic attacks, intermittent claudication, peripheral bypass or stent, and amputation; ${ }^{\mathrm{C} C o m p o s i t e ~ s c o r e ~ o f ~}$ occurrence of microalbuminuria, renal impairment (serum creatinine $>1.5 \mathrm{mg} / \mathrm{dL}$ ), diabetic nephropathy, and proteinuria; (hemorrhages, exudates, papilloedema), C-reactive protein $\geq 1 \mathrm{mg} / \mathrm{dL}$, and family history of premature cardiovascular disease (at age $<55$ for men, $<65$ for women).

Abbreviations: DBP, diastolic blood pressure; SBP, systolic blood pressure. 


\section{Modeling}

Logistic regression modelling ${ }^{15}$ was used to model uncontrolled SBP, DBP, and SBP/DBP at 90 days. In this analysis, it was assumed that predictive validity could be inferred if four conditions were met. First, statistically significant logistic regression models could be fit using vulnerability profiles to predict uncontrolled BP. Second, odds ratios (ORs) for uncontrolled BP as a function of being highly vulnerable exceed 1.00 , and the associated $95 \%$ confidence intervals (CIs) do not cross 1.00. Third, each model is sufficiently sensitive to correctly classify patients' BP control status as indicated by the correct classification rate (CCR). Fourth, given the availability of data on initial BP control, and BP control after valsartan treatment, the first three conditions are met for first-line, second-line, and first-or-second-line antihypertensive treatment.

\section{Results}

Table 2 summarizes the logistic regressions for uncontrolled SBP, DBP, and combined SBP/DBP for HVC patients. All models were significant at $P<0.001$. All ORs for $\mathrm{HVC}$ were statistically greater than 1.00 and none had $95 \%$ CIs crossing 1.00 (all $P<0.001$ ). CCRs for uncontrolled BP after first-, second-, or either treatment were $91.1 \%, 61.2 \%$, and $93.5 \%$ for SBP; $74.5 \%, 65.8 \%$, and $76.7 \%$ for DBP; and $92.8 \%$, $65.3 \%$, and $94.6 \%$ for combined SBP/DBP.

\section{Discussion}

We accurately identified patients who had been diagnosed relatively late and with more severe hypertension (later); those weighing more, drinking more, and exercising less (lazier); and those who present with greater general, cardiovascular,

Table 2 Logistic regression results of uncontrolled blood pressure at follow-up as a function of membership in the high-vulnerability cluster

\begin{tabular}{llll}
\hline & OR & $\mathbf{9 5 \%} \mathbf{C l}$ & CCR \\
\hline After first-line treatment & & & \\
Uncontrolled SBP & 1.789 & 1.338 to 2.391 & $91.1 \%$ \\
Uncontrolled DBP & 1.443 & 1.200 to 1.711 & $74.5 \%$ \\
Uncontrolled SBP/DBP & 1.550 & 1.137 to 2.115 & $92.8 \%$ \\
After second-line treatment with valsartan & & \\
Uncontrolled SBP & 1.562 & 1.33 I to 1.833 & $61.2 \%$ \\
Uncontrolled DBP & 1.534 & 1.309 to 1.797 & $65.8 \%$ \\
Uncontrolled SBP/DBP & 1.717 & 1.455 to 2.028 & $65.3 \%$ \\
After either first- or second-line treatment & & \\
Uncontrolled SBP & 2.071 & 1.459 to 2.940 & $93.5 \%$ \\
Uncontrolled DBP & 1.553 & 1.290 to 1.869 & $76.7 \%$ \\
Uncontrolled SBP/DBP & 1.898 & 1.340 to 2.761 & $94.6 \%$ \\
\hline
\end{tabular}

Abbreviations: $\mathrm{CCR}$, correct classification rate; $\mathrm{Cl}$, confidence interval; $\mathrm{DBP}$, diastolic blood pressure; OR, odds ratio; SBP, systolic blood pressure. and renal/endocrine risk and have a family history of premature cardiovascular disease (unluckier) as being highlyvulnerable to poor BP control. Again, the profile's labelling is only with clinical mnemonics in mind and without any derogatory intent.

Being a highly-vulnerable patient was significantly associated with uncontrolled BP after first-line antihypertensive therapy with excellent predictive power. Thus, if patients presenting in the clinic fit the general profile of "later, lazier, and unluckier", this should serve as a strong warning signal to clinicians that this subgroup of patients is much less likely to meet BP targets after first-line therapy, leaving them more prone to end-organ damage and requiring transition to second-line treatment. Likewise, this general profile was helpful in identifying patients who were less likely to achieve BP control after either first- or second-line treatment, with minimally higher predictive validity compared to first-line therapy alone.

Many patients with hypertension are started on monotherapy and often require two or more antihypertensive agents in combination. ${ }^{13,14}$ Being a HVC patient increased the odds of having uncontrolled BP after second-line treatment, but with somewhat less predictive power. This may be due to the fact that second-line agents are started on patients who may be resistant to antihypertensive treatment, or patients in whom other factors, such as comorbid conditions or poor adherence, have not been identified and/or sufficiently addressed. This may be due to the fact that by switching from one antihypertensive drug (first-line) to another (second-line) involved a change in antihypertensive class. In Belgium, thiazide diuretics are often prescribed as first-line antihypertensive drugs; thus, changing to valsartan often involved a switch from an antivolume to an antiresistance drug. ${ }^{16}$ Fitting the general profile of "later, lazier, and unluckier", however, should serve as a caution for clinicians that this subgroup of patients is also less likely to meet BP targets after secondline treatment.

\section{Conclusion}

With this additional evidence of the predictive validity of vulnerability profiling on BP control, clinicians have a more intuitive but empirically-grounded assessment of patient risk. The general profile of "later, lazier, unluckier" is helpful in identifying patients at greater risk for poor BP control.

\section{Disclosure}

Drs Abraham, Lee, Song, and MacDonald are employees of Matrix45 and by company policy are prohibited from owning equity in client organizations. Dr Van der Niepen has consulted with and received research grants and contracts 
from Novartis Pharma. Drs Vancayzeele and Brié and Ms Hermans are employees of Novartis Pharma.

\section{References}

1. D'Agostino RBS, Grundy S, Sullivan LM, Wilson P. Validation of the Framingham coronary heart disease prediction scores: results of a multiple ethnic groups investigation. JAMA. 2001;286:180-187.

2. Conroy RM, Pyorala K, Fitzgerald AP, et al. Estimation of ten-year risk of fatal cardiovascular disease in Europe: the SCORE project. Eur Heart J. 2003;24:987-1003

3. Berlowitz DR, Ash AS, Hickey EC, et al. Inadequate management of blood pressure in a hypertensive population. New Engl J Med. 1998;339: 1957-1963.

4. Fagard RH, Van den Enden M, Leeman M, Warling X. Survey on treatment of hypertension and implementation of World Health Organization/ International Society of Hypertension risk stratification in primary care in Belgium. J Hypertens. 2002;20:1297-1302.

5. Van der Niepen P, Giot C, van de Borne P. Prevalence of isolated uncontrolled systolic blood pressure among treated hypertensive patients in primary care in Belgium. Results of the I-inSYST survey. J Hypertens. 2008;26:2057-2063.

6. Ong KL, Cheung BM, Man YB, Lau CP, Lam KS. Prevalence, awareness, treatment, and control of hypertension among United States adults 1999-2004. Hypertension. 2007;49:69-75.

7. Staessen JA, Wang JG, Thijs L. Cardiovascular prevention and blood pressure reduction: a quantitative overview updated until 2003 March 1. J Hypertens. 2003;21:1055-1076.
8. Kannel WB. Elevated systolic blood pressure as a cardiovascular risk factor. Am J Cardiol. 2000;85:251-255.

9. Van der Niepen P, Woestenburg A, Brié H, et al. Effectiveness vs efficacy of valsartan for treatment of hypertension: patient profiling and hierarchical modeling of determinants and outcomes. Ann Pharmacother. 2009;43:849-861.

10. Tan PN, Steinbach M, Kumar V. Data Mining. Boston, MA: AddisonWesley; 2005.

11. Aldenderfer MS, Blashfield RK. Cluster Analysis. Thousand Oaks, CA: Sage; 1984.

12. Everitt BS, Landau B, Leese M. Cluster analysis. 2001. 4th ed. New York: Oxford University Press.

13. Chobanian AV, Bakris GL, Black HR, et al. The Seventh Report of the Joint National Committee on prevention, detection, evaluation, and treatment of high blood pressure: the JNC-7 report. J Am Med Assoc. 2003;289:2560-2572.

14. Guidelines Committee. 2003 European Society of Hypertension European Society of Cardiology guidelines for the management of arterial hypertension. J Hypertens. 2003;21:1011-1053.

15. Kleinbaum DG, Klein M. Logistic regression. 2005. 2nd ed. New York, NY: Springer-Verlag.

16. Laragh JH. Abstract, closing summary, and table of contents for Laragh's 25 lessons in pathophysiology and 12 clinical pearls for treating hypertension. Am J Hypertens. 2001;14:1173-1174.
International Journal of General Medicine

\section{Publish your work in this journal}

The International Journal of General Medicine is an international, peer-reviewed open-access journal that focuses on general and internal medicine, pathogenesis, epidemiology, diagnosis, monitoring and treatment protocols. The journal is characterized by the rapid reporting of reviews, original research and clinical studies across all disease areas.

\section{Dovepress}

A key focus is the elucidation of disease processes and management protocols resulting in improved outcomes for the patient.The manuscript management system is completely online and includes a very quick and fair peer-review system. Visit http://www.dovepress.com/ testimonials.php to read real quotes from published authors. 